\title{
Neurocognitive Markers of Suicidal Ideation in Patients with Anxiety and
}

\section{Mood Disorders}

Vilma Liaugaudaite $^{\mathrm{a} *}$ Naomi A. Fineberg ${ }^{\mathrm{b}, \mathrm{c}}$, Aurelija Podlipskyte ${ }^{\mathrm{a}}$, Julija

Gečaitė $^{\mathrm{a}}$, Alicja Juškienè ${ }^{\mathrm{a}}$, Narseta Mickuviene ${ }^{\mathrm{a}}$, Julius Burkauskas ${ }^{\mathrm{a}}$

${ }^{a}$ Laboratory of Behavioral Medicine, Neuroscience Institute, Lithuanian University of Health Sciences, Palanga, Lithuania

${ }^{b}$ National Obsessive Compulsive Disorders Specialist Service, Hertfordshire Partnership

University NHS Foundation Trust, Welwyn Garden City, UK

${ }^{c}$ University of Hertfordshire, UK

Vilma Liaugaudaite, MSC (corresponding author)

$\mathrm{PhD}$ student, Junior Researcher, Public Health Specialist

Laboratory of Behavioral Medicine, Neuroscience Institute, Lithuanian University of Health Sciences

Vyduno al. 4, LT-00135 Palanga, Lithuania

Email: vilma.liaugaudaite@1smuni.lt

Telephone: +370 46030012

Fax: +370 46030014 


\title{
Neurocognitive Markers of Suicidal Ideation in Patients with Anxiety and Mood Disorders
}

\begin{abstract}
Objective: This study aims at identifying associations between cognitive function and suicidal ideation in the sample of patients with anxiety and mood disorders (AMD).

Methods: In sum, 186 (age $=39 \pm 12.3$ years; 142 [76.3\%] females) patients with AMD were enrolled in the study. Assessment included evaluation of socio-demographic information, medication use, anxiety and depression symptoms. Cognitive tests included measures of psychomotor performance and incidental learning using the Digit Symbol Test. Trail Making Tests respectively measured perceptual speed, task-switching and executive control. Additionally, 21 patients completed tests from the Cambridge Automated Neuropsychological Test Battery measuring set shifting (Interdimensional/extradimensional set-shift), executive planning (Stockings of Cambridge), and decision making (Cambridge Gamble Task [CGT]).

Results: Almost half $(45.0 \%, \mathrm{n}=86)$ of the study sample patients had experienced suicidal ideations. In multivariable regression analysis, suicidal ideation was associated with a greater overall proportion of bet and risk taking on the CGT task $(\beta=.726, p=.010$ and $\beta=.634, p=$ .019), when controlling for socio-demographic characteristics, medication use, anxiety and depression symptoms.

Conclusions: Outpatients with AMD and suicidal ideation could be distinguished by the presence of cognitive deficits in the executive function domain, particularly in impulse-control and risk taking.
\end{abstract}

Keywords: suicidal ideation, neurocognitive markers, anxiety and mood disorders. 


\section{Background}

Patients with anxiety and mood disorders (AMD) frequently experience suicidal ideation in the week prior to attempting suicide (Nock, Hwang, Sampson, \& Kessler, 2010). Patients with AMD also show cognitive deficits, with impairments in executive functioning domains being the most prominent (Pu, Setoyama, \& Noda, 2017). Recent studies have focused on finding factors that differentiate those who think about suicide from those who make a suicide attempt (Wetherall et al., 2018). However, the most up to date review on the topic has proposed to separately consider neurocognitive predictors of ideation (Boaz Y Saffer \& Klonsky, 2018) as this would allow to capture a more detailed neuropsychological profile of this particular group. A better understanding of cognitive schemas in patients who have suicidal ideation may help clinicians to indicate the need for emergency care and early intervention.

Our study aimed at identifying associations between cognitive functioning and suicidal ideation in a sample of patients with AMD, by using specific neurocognitive assessment with relevant tests of executive function including impulsive decision-making, executive planning and attentional set-shifting.

\section{Methods}

This cross-sectional study included 186 (age $=39 \pm 12.3$ years; 142 [76.3\%] females) consecutive outpatients with AMD attending the Stress Disorders Clinic in Palanga, Lithuania. Socio-demographic and clinical information including diagnosis and suicidal ideation, as defined by The Mini International Neuropsychiatric Interview (MINI) (Sheehan et al., 1998), were collected. The Hamilton 14-item Anxiety (HAM-A) and 17-item Depression (HAM-D) Rating Scales were used to evaluate symptoms of anxiety and depression (Hamilton, 1959, 1960; Zimmerman et al., 2013). In this study population, the HAM-A (Cronbach's $\alpha=.900$ ) and HAM-D (Cronbach's $\alpha=.833$ ) had shown a good internal reliability. Psychomotor performance and incidental learning were assessed using the Digit Symbol Test (Wechsler, 1981). Trail Making Tests A and B respectively measured perceptual speed, task-switching and executive control (Strauss, Sherman, \& Spreen, 2006).

Additionally, 21 consecutive patient with AMD completed tests from the Cambridge Automated Neuropsychological Test Battery CANTAB® (CANTAB®, 2018) including tests of set-shifting (Interdimensional/extradimensional set-shift tasks [ID/ED]), executive planning (Stockings of Cambridge [SOC]), and decision making (Cambridge Gamble Task [CGT]). Non-completers did not differ significantly from completers in socio-demographic characteristics, medication use, and depressive/anxiety symptom severity (all p's > .05). 
Two-tailed Student's t-test, Fisher's $\chi 2$ or Mann-Whitney U tests were applied to compare socio-demographic, clinical, anxiety, depression as well as cognitive function characteristics in AMD patients with and without suicidal ideation. We used Benjamini-Hochberg adjustment for multiple comparisons in the cognitive function domain, setting a critical value for a false discovery rate of .10 .

Next, we sought to investigate if discovered differences in cognitive functioning testing results, as a function of suicidal ideation, were independent from socio-demographic characteristics, medication use, and depressive/anxiety symptom severity. We employed multivariable regression analysis with a specific cognitive domain as the dependent variable and the presence of suicidal ideation as an independent factor adjusting for age (in years), gender (men [1] or women [2]), education status (up to 8 years [1], high school [2] or college/university degree [3]), other currently used medications, such as antidepressants, tranquilizers, mood stabilizers and neuroleptics (use [1] or non-use [0]), and depressive (HAM-D score) and anxiety symptom severity (HAM-A score). All statistical analyses were conducted using the SPSS 17.0 for Windows.

\section{Results}

A total of 186 patients were included in the final analysis. In this sample group, approximately $21.5 \%(\mathrm{n}=40)$ patients had been diagnosed with one mood disorder, $28.5 \%(\mathrm{n}=53)$ had been diagnosed with one anxiety disorder, and 50.0\% $(\mathrm{n}=93)$ patients had been diagnosed with AMD. Almost half of patients included $(45.7 \%, \mathrm{n}=85)$ had suicidal ideations (Table 1). Scores on the CGT overall proportion bet and the CGT risk taking test were found to be significantly higher among patients with suicidal ideation as compared to patients without suicidal ideation (.57[.51-.70] vs. .46[.41-.51], $\mathrm{U}=17.0, \mathrm{p}=.007 ; .65[.55-.75]$ vs. .51[.49-.54], $\mathrm{U}=12.0, \mathrm{p}=$ $.002)$. There were no other significant differences for neurocognitive test scores among those with and without suicidal ideation (all p's > .05).

Next we evaluated if performance on the CGT overall proportion bet and the CGT risk taking as function of suicidal ideation were independent from evaluated socio-demographic characteristics, medication use, and depressive/anxiety symptoms. Furthermore, in a multivariable regression analysis, suicidal ideation was associated with greater overall proportion bet and risk taking on the CGT task $(\beta=.726, p=.010$ and $\beta=.634, p=.019)$ after adjusting for age, gender, education, currently used medication, depressive symptoms and anxiety symptoms. 


\section{Discussion}

Our study results confirmed previous findings that suicidal ideation is associated with impaired cognitive performance and poor decision-making (Marzuk, Hartwell, Leon, \& Portera, 2005; Onat et al., 2018; B. Y. Saffer \& Klonsky, 2017; Westheide et al., 2008).

Neurocognitive functions have received little consideration in the suicide risk assessment of patients with AMD. A neurocognitive model of suicidal behaviour suggests that the alteration of specific cognitive functions may facilitate the development of a suicidal crisis during stressful circumstances, independently of psychiatric comorbidity (Richard-Devantoy, Ding, Lepage, Turecki, \& Jollant, 2016).

In this study, markers of impulsive decision making, but not of executive planning or setshifting, differentiated those with suicidal ideation, which is reflective of previous research findings in psychiatric samples (Gvion \& Apter, 2011). However, we did not observe any differences between groups in other areas of neurocognitive functioning.

A recent meta-analysis by Richard-Devantoy and colleagues claim that even though TMT is adequate and widely used as a test to measure cognitive deficits in psychiatric patients with suicidal ideation, TMT is still more closely related to symptoms of mood disorders than to suicidal behaviour (Richard-Devantoy et al., 2016). This may explain our study results of nonsignificant relationship between TMT and suicidal ideation. A recent review has pointed out that ideators differed most from non-suicidal individuals on measures of processing speed, global executive functions, and global cognitive function (Boaz Y Saffer \& Klonsky, 2018). Given the fact that we used MINI to identify ideators we had a lack of information about frequency and severity of suicide ideation. We could expect that mild and severe forms of ideation might influence differently neurocognitive function. In addition to this, cross-sectional nature of the study precludes us from making causal implications. Without knowing the temporal sequence of events, it is only a hypothesis whether neurocognitive differences precede suicide ideation. For example, a large prospective study of U.S. soldiers found that global cognitive function impairment predicted suicide ideation (Naifeh et al., 2017). Thus, future studies should longitudinally assess cognitive predictors of ideation and suicide ideation frequency and severity to obtain more detailed profile of neurocognitive function of those with suicidal ideation.

Cognitive deficits may constitute relevant intervention targets for identifying or treating suicidality. Our results suggest that testing for neurocognitive biomarkers of suicide ideation risk may complement clinical assessment in patients with AMD. 
The strengths of our study include well-validated scales, as well as modern instruments for measuring neurocognitive markers. Although this research was carefully prepared, there are several limitations to be considered when interpreting results. First, there was a lack of prospective observation and a relatively small sample size. Second, the generalizability of these results may be limited as this study was conducted in a small sample of AMD patients at a single unit.

\section{Conclusion}

Our study indicates that AMD patients with suicidal ideation could be distinguished by the presence of cognitive deficits in the executive function domain, particularly in impulse-control and risk taking. Cognitive deficits are important therapeutic targets and may allow clinicians to evaluate the potential of the CANTAB to serve as a cognitive biomarker of the suicide ideation risk in patients with AMD. Further longitudinal studies examining other cognitive functions that could have an impact on suicidal risk in patients with AMD are warranted. 


\section{References}

CANTAB®. (2018). [Cognitive assessment software]. Cambridge Cognition (2018). All rights reserved. www.cantab.com.

Gvion, Y., \& Apter, A. (2011). Aggression, impulsivity, and suicide behavior: a review of the literature. Arch Suicide Res, 15(2), 93-112. doi: 10.1080/13811118.2011.565265

Marzuk, P. M., Hartwell, N., Leon, A. C., \& Portera, L. (2005). Executive functioning in depressed patients with suicidal ideation. Acta Psychiatr Scand, 112(4), 294-301. doi: 10.1111/j.16000447.2005.00585.x

Naifeh, J. A., Nock, M. K., Ursano, R. J., Vegella, P. L., Aliaga, P. A., Fullerton, C. S., . . Army, S. C. (2017). Neurocognitive Function and Suicide in U.S. Army Soldiers. Suicide \& life-threatening behavior, 47(5), 589-602. doi: 10.1111/sltb.12307

Nock, M. K., Hwang, I., Sampson, N. A., \& Kessler, R. C. (2010). Mental disorders, comorbidity and suicidal behavior: results from the National Comorbidity Survey Replication. Mol Psychiatry, 15(8), 868-876. doi: 10.1038/mp.2009.29

Onat, M., İnal Emiroğlu, N., Baykara, B., Özerdem, A., Özyurt, G., Öztürk, Y., . . Pekcanlar Akay, A. (2018). Executive functions and impulsivity in suicide attempter adolescents with major depressive disorder. Psychiatry and Clinical Psychopharmacology, 1-8. doi: 10.1080/24750573.2018.1541647

$\mathrm{Pu}, \mathrm{S}$., Setoyama, S., \& Noda, T. (2017). Association between cognitive deficits and suicidal ideation in patients with major depressive disorder. Sci Rep, 7(1), 11637. doi: 10.1038/s41598-017$12142-8$

Richard-Devantoy, S., Ding, Y., Lepage, M., Turecki, G., \& Jollant, F. (2016). Cognitive inhibition in depression and suicidal behavior: a neuroimaging study. Psychol Med, 46(5), 933-944. doi: $10.1017 / \mathrm{s} 0033291715002421$

Saffer, B. Y., \& Klonsky, E. D. (2017). The Relationship of Self-reported Executive Functioning to Suicide Ideation and Attempts: Findings from a Large U.S.-based Online Sample. Arch Suicide Res, 21(4), 577-594. doi: 10.1080/13811118.2016.1211042

Saffer, B. Y., \& Klonsky, E. D. (2018). Do neurocognitive abilities distinguish suicide attempters from suicide ideators? A systematic review of an emerging research area. Clinical Psychology: Science and Practice, 25(1), e12227.

Sheehan, D. V., Lecrubier, Y., Sheehan, K. H., Amorim, P., Janavs, J., Weiller, E., . . Dunbar, G. C. (1998). The Mini-International Neuropsychiatric Interview (M.I.N.I.): the development and validation of a structured diagnostic psychiatric interview for DSM-IV and ICD-10. J Clin Psychiatry, 59 Suppl 20, 22-33;quiz 34-57.

Strauss, E., Sherman, E., \& Spreen, O. (2006). A compendium of of neuropsychological tests: Administration, norms, and commentary. New York: Oxford University Press.

Wechsler, D. (1981). The Wechsler Adult Intelligence Scale-Revised (WAIS-R). San Antonio, Texas: Psychological Corporation.

Westheide, J., Quednow, B. B., Kuhn, K. U., Hoppe, C., Cooper-Mahkorn, D., Hawellek, B., .. W Wagner, M. (2008). Executive performance of depressed suicide attempters: the role of suicidal ideation. Eur Arch Psychiatry Clin Neurosci, 258(7), 414-421. doi: 10.1007/s00406-008-0811-1

Wetherall, K., Cleare, S., Eschle, S., Ferguson, E., O'Connor, D. B., O'Carroll, R. E., \& O'Connor, R. C. (2018). From ideation to action: Differentiating between those who think about suicide and those who attempt suicide in a national study of young adults. $J$ Affect Disord, 241, 475-483. doi: 10.1016/j.jad.2018.07.074 


\section{Acknowledgements}

This study was partially funded by an International Brain Research Organization (IBRO) PanEuropean Regional Committee (IBRO-PERC) In Europe Short Stay Grant received by Dr. Julius Burkauskas.

\section{Conflict of interest}

Dr. Naomi A. Fineberg who in the past several years, has received research support from Lundbeck, Glaxo-SmithKline, European College of Neuropsychopharmacology (ECNP), Servier, Cephalon, Astra Zeneca, Medical Research Council (UK), National Institute for Health Research, Wellcome Foundation, University of Hertfordshire, EU (FP7), Shire. Dr. Fineberg has received honoraria for lectures at scientific meetings from Abbott, Otsuka, Lundbeck, Servier, Astra Zeneca, Jazz pharmaceuticals, Bristol Myers Squibb, UK College of Mental Health Pharmacists, British Association for Psychopharmacology (BAP), Wiley. Dr Fineberg has received financial support to attend scientific meetings from RANZCP, Shire, Janssen, Lundbeck, Servier, Novartis, Bristol Myers Squibb, Cephalon, International College of Obsessive-Compulsive Spectrum Disorders, International Society for Behavioural Addiction, CINP, IFMAD, ECNP, BAP, World Health Organization, Royal College of Psychiatrists. Dr. Fineberg has received financial royalties for publications from Oxford University Press and payment for editorial duties from Taylor and Francis.

Julija Gecaite works as a consultant at FACITtrans. Dr. Julius Burkauskas has served as a consultant at Cogstate, Ltd. Remaining authors declare no conflict of interest. 
Table 1. Socio-demographic and clinical characteristics of the patients.

\begin{tabular}{|c|c|c|c|c|}
\hline & $\begin{array}{l}\text { Total } \\
\mathrm{N}=186\end{array}$ & $\begin{array}{l}\text { Without SI } \\
\mathrm{N}=101\end{array}$ & $\begin{array}{l}\text { With SI } \\
\mathbf{N}=85\end{array}$ & $\begin{array}{l}\text { p } \\
\text { value }\end{array}$ \\
\hline Age, mean \pm SD & $38.9 \pm 12.3$ & $40.1 \pm 11.0$ & $37.5 \pm 13.6$ & .169 \\
\hline \multicolumn{5}{|l|}{ Gender, n (\%) } \\
\hline Men & $44(23.7)$ & $25(24.8)$ & $19(22.4)$ & .732 \\
\hline Women & $142(76.3)$ & $76(75.2)$ & $66(77.6)$ & \\
\hline \multicolumn{5}{|l|}{ Education, n (\%) } \\
\hline Secondary & $65(34.9)$ & $31(30.7)$ & $34(40.0)$ & .394 \\
\hline College & $23(12.4)$ & $14(13.9)$ & $9(10.6)$ & \\
\hline University & $98(52.7)$ & $56(55.4)$ & $42(49.4)$ & \\
\hline \multicolumn{5}{|l|}{ Diagnosis, n (\%) } \\
\hline Major depression disorder & $40(21.5)$ & $21(20.8)$ & $19(22.4)$ & $<.001$ \\
\hline Anxiety disorder & $53(28.5)$ & $41(40.6)$ & $12(14.1)$ & \\
\hline Mood and anxiety disorder & $93(50.0)$ & $39(38.6)$ & $54(63.5)$ & \\
\hline \multicolumn{5}{|l|}{ Current medication use, $n(\%)$} \\
\hline Antidepressants & 137 (73.7) & $68(67.3)$ & $69(81.2)$ & .033 \\
\hline Tranquilizers & $76(40.9)$ & $38(37.6)$ & $38(44.7)$ & .328 \\
\hline Mood stabilizers & $7(3.8)$ & $1(1.0)$ & $6(7.1)$ & .030 \\
\hline Neuroleptics & $47(25.3)$ & $22(21.8)$ & $25(29.4)$ & .233 \\
\hline Obesity $($ BMI >30 kg/m²), n(\%) & $28(15.6)$ & $12(12.2)$ & $16(19.8)$ & .169 \\
\hline History of smoking, $n(\%)$ & $49(26.3)$ & $20(19.8)$ & $29(34.1)$ & .027 \\
\hline Depression Hamilton, mean \pm SD & $14.9 \pm 7.3$ & $13.1 \pm 6.5$ & $17.1 \pm 7.7$ & $<.001$ \\
\hline Anxiety Hamilton, mean \pm SD & $24.0 \pm 9.9$ & $22.3 \pm 9.5$ & $26.2 \pm 9.9$ & .007 \\
\hline \multicolumn{5}{|l|}{ Digit Symbol Test, mean \pm SD } \\
\hline Raw score & $46.4 \pm 10.9$ & $46.7 \pm 9.9$ & $46.0 \pm 12.0$ & .709 \\
\hline Pairs recalled correctly & $5.3 \pm 2.6$ & $5.3 \pm 2.6$ & $5.4 \pm 2.5$ & .660 \\
\hline Digit Symbol Test time (s) & $138.9 \pm 38.5$ & $137.2 \pm 35.2$ & $140.9 \pm 42.3$ & .518 \\
\hline \multicolumn{5}{|l|}{ Trail Making Test, mean \pm SD } \\
\hline Test A Time (s) & $29.2 \pm 11.0$ & $28.4 \pm 9.2$ & $30.3 \pm 12.8$ & .267 \\
\hline Test B Time (s) & $75.1 \pm 32.3$ & $76.0 \pm 29.7$ & $74.1 \pm 35.4$ & .695 \\
\hline \multicolumn{5}{|c|}{$\begin{array}{l}\text { Cambridge Gambling Task, median (IQR) } \\
\mathrm{N}=21\end{array}$} \\
\hline Deliberation time (ms) & $2711(2217-3157)$ & $2627(2225-3122)$ & $2743(2199-3286)$ & .573 \\
\hline Overall proportion bet & $.51(.46-.68)$ & $.46(.41-.51)$ & $.57(.51-.70)$ & $.007 *$ \\
\hline Quality of decision making & $.92(.87-.96)$ & $.92(.74-.96)$ & $.92(.88-.97)$ & .481 \\
\hline Risk adjustment & $.73(-0.02-1.3)$ & $.72(.11-1.4)$ & $.73(-.04-1.1)$ & .526 \\
\hline Risk taking & $.55(.51-.69)$ & $.51(.49-.54)$ & $.65(.55-.75)$ & $.002 *$ \\
\hline \multicolumn{5}{|c|}{$\begin{array}{l}\text { Intradimensional/extradimensional set-shift } \\
\text { tasks, median (IQR) }\end{array}$} \\
\hline Total errors adjusted & $14.0(10.0-55.5)$ & $13.5(8.0-55.8)$ & $17.0(11.0-56.0)$ & .378 \\
\hline Stages completed & $9.0(8.0-9.0)$ & $9.0(7.0-9.0)$ & $9.0(9.0-9.0)$ & .535 \\
\hline \multicolumn{5}{|l|}{ Stockings of Cambridge Task } \\
\hline Mean moves 5 moves & $6.8(5.6-8.3)$ & $7.1(5.8-8.6)$ & $6.8(5.5-7.5)$ & .396 \\
\hline
\end{tabular}

Student's t or Mann-Whitney U test was used for continuous variables and the $\chi 2$ test or Fisher's Exact test for categorical variables.

* Statistically significant (significance of $\mathrm{p}$ values in cognitive functioning domains are adjusted to Benjamini-Hochberg correction for multiple comparisons) 0.10 . 